\title{
Papers
}

\section{0 years' follow up of randomised studies of adjuvant CMF in operable breast cancer: cohort study}

Gianni Bonadonna, Angela Moliterni, Milvia Zambetti, Maria Grazia Daidone, Silvana Pilotti, Luca Gianni, Pinuccia Valagussa

\begin{abstract}
Objective To assess the long term effectiveness of adjuvant treatment with cyclophosphamide, methotrexate, and fluorouracil (CMF) in patients with operable breast cancer at risk of relapse, on the basis of three successive randomised trials and one observational study conducted from June 1973 to December 1980.

Design Cohort study.

Setting Istituto Nazionale Tumori in Milan, Italy.

Main outcome measures Relapse free and overall survival, measured by univariate and multivariate analyses.

Results After a median follow up of 28.5 years for the initial study, adjuvant CMF was found to reduce the relative risk of relapse significantly (hazard ratio $0.71,95 \%$ confidence interval 0.56 to $0.91, \mathrm{P}=0.005)$ and death $(0.79,0.63$ to $0.98, \mathrm{P}=0.04)$. Administration of CMF for 12 cycles does not seem superior to a shorter administration of six cycles. In the node negative and oestrogen receptor negative trial, intravenous CMF significantly reduced the relative risk of relapse of disease $(0.65,0.47$ to 0.90 , $\mathrm{P}=0.009)$ and death $(0.65,0.47$ to $0.92, \mathrm{P}=0.01)$ at a median follow up of 20 years.

Conclusions When delivered optimally, CMF benefits patients at risk of relapse of distant disease without evidence of detrimental effects in any of the examined subgroups.
\end{abstract}

\section{Introduction}

In 1975 we presented our first report on the efficacy of cyclophosphamide, methotrexate, and fluorouracil (CMF) as adjuvant treatment for node positive breast cancer. ${ }^{12}$ These results, along with those reported in a similar population of patients by the National Surgical Adjuvant Breast and Bowel Project, ${ }^{3}$ raised hopes that chemotherapy could have a more central role in the primary management of this common cancer. The worldwide overview confirmed that, when the long term benefits of this treatment modality are balanced against its risks, adjuvant chemotherapy can be worth while in many patients with breast cancer. ${ }^{4}$ Nevertheless, questions have been raised in the past years concerning the true effectiveness of adjuvant CMF for specific subgroups of patients. ${ }^{56}$ We report the results of 30 years of experience with adjuvant CMF in a series of successive clinical trials.

\section{Methods}

The study designs of the randomised trials were reported earlier $^{2-9}$ and are summarised in table 1 . The first randomised study started in June 1973, and by September 1975, 386 eligible patients with node positive breast cancer had been allocated to receive either no further treatment after radical mastectomy or adjuvant CMF for 12 cycles. $^{27}$ The second randomised trial started in September 1975, and initially premenopausal and postmenopausal women were allocated to receive either 12 or six cycles of postoperative CMF. In November 1976, however, random enrolment of postmenopausal women was discontinued, and the study was limited to premenopausal patients; the recruitment was closed in May 1978, and the sample included 324 eligible, node positive, premenopausal women. An observational study between May 1978 and October 1980 included 220 premenopausal patients with node positive breast cancer, all scheduled to receive 12 cycles of CMF. The fourth trial, between December 1980 and October 1985, included 90 women with node negative and oestrogen receptor negative tumours, who were randomised to either no systemic treatment after surgery or 12 cycles of intravenous CMF given every three weeks. ${ }^{9}$

The study populations consisted of patients admitted to the Istituto Nazionale Tumori in Milan, Italy. All women who had had surgery (radical mastectomy or conservative surgery and full axillary clearance) for unilateral breast cancer were considered for inclusion in the studies if they had histological evidence that one or more axillary nodes were affected (first three studies) or if they had histologically negative axillary nodes and oestrogen receptor negative tumours (third study).

Patients with locally advanced or metastatic disease, those with a history of previous cancer, and those with concomitant severe non-malignant systemic disease were not eligible.

With the exception of the randomised trial investigating oestrogen receptor negative tumours (the third trial), assessment of the hormone receptors was not mandatory and was done only retrospectively, by using the dextran coated charcoal technique. This was done in all but the first study, in which they were assessed on immunohistochemistry. According to Italian rules at the time, all patients had to give verbal informed consent before being enrolled into each of the studies.

\section{Adjuvant treatment}

In patients with node positive breast cancer, CMF consisted of cyclophosphamide (100 mg/m² orally from day 1 to 14 ), methotrexate $\left(40 \mathrm{mg} / \mathrm{m}^{2}\right.$ intravenously on days 1 and 8 ), and fluorouracil $\left(600 \mathrm{mg} / \mathrm{m}^{2}\right.$ intravenously on days 1 and 8 ), repeated every four weeks for either six or 12 cycles. $^{2}$ In this subset of patients, women older than 60 were to receive reduced doses of methotrexate $\left(30 \mathrm{mg} / \mathrm{m}^{2}\right)$ and fluorouracil $(400$ $\left.\mathrm{mg} / \mathrm{m}^{2}\right)$. 


\begin{tabular}{|c|c|c|c|c|}
\hline Enrolment period & Study design & Eligible patients & Intervention & No of patients \\
\hline June 1973 to September 1975 & Randomised controlled trial & $\begin{array}{l}\text { Node positive, premenopausal, and } \\
\text { postmenopausal }\end{array}$ & Surgery $v$ CMF for 12 cycles & 179 v 207 \\
\hline September 1975 to May 1978 & Randomised controlled trial & Node positive, premenopausal & $\begin{array}{c}\text { CMF for } 12 \text { cycles } v \text { CMF for } 6 \\
\text { cycles }\end{array}$ & $160 \vee 164$ \\
\hline May 1978 to October 1980 & Observational study & Node positive, premenopausal & CMF for 12 cycles & 220 \\
\hline December 1980 to October 1985 & Randomised controlled trial & $\begin{array}{c}\text { Node negative and oestrogen receptor } \\
\text { negative, premenopausal, and } \\
\text { postmenopausal }\end{array}$ & $\begin{array}{c}\text { Surgery } v \text { intravenous CMF for } 12 \\
\text { cycles }\end{array}$ & $45 \vee 45$ \\
\hline
\end{tabular}

In the third study, 12 cycles of cyclophosphamide $(600$ $\left.\mathrm{mg} / \mathrm{m}^{2}\right)$, methotrexate $\left(40 \mathrm{mg} / \mathrm{m}^{2}\right)$, and fluorouracil $(600$ $\mathrm{mg} / \mathrm{m}^{2}$ ) were given intravenously on day 1 and repeated three weeks later. No dose reductions for older patients were planned. ${ }^{9}$

In all studies, treatment with CMF was started two to four weeks after surgery. No other adjuvant treatments, in particular no endocrine treatment, were allowed, with the exception of breast irradiation for patients who had had conservative surgery. Breast irradiation (40 Gray (Gy) plus a boost of $10 \mathrm{~Gy}$ in four to six weeks) had to be initiated within six to eight weeks from surgery and was administered alongside CMF in women allocated to receive adjuvant chemotherapy.

\section{Study variables}

Details on baseline studies and follow up programmes are reported elsewhere..$^{2-9}$ Before surgery, all patients had a complete physical examination and were investigated by radiological and biochemical techniques. In the absence of symptoms, women had a complete physical examination every three months during the first year, every six months for the next four years, and every 12 months for the following 10 years. Biochemical tests and radiological studies were done every six to eight months during the first five years, and once a year thereafter. Liver ultrasonography was undertaken only if clinical or biochemical findings were suspicious. Mammography, bilateral in patients who had had conservative surgery, was scheduled once a year. After the 15th year of follow up, examinations were set for every 12 to 24 months, and when these were not done in the outpatient clinic of the institute, contact was periodically maintained with the patients and their family doctors. Patients with suspicious or controversial findings were examined more often. We considered treatment to have failed when the first evidence of new manifestations of disease in locoregional areas (including ipsilateral supraclavicular adenopathy), distant sites, the contralateral breast, or any combination of these sites was documented. We considered neither second primary cancers nor deaths owing to causes other than breast cancer treatment failures.

\section{Statistical analysis}

We calculated relapse free survival from the date of surgery to the first documented evidence of treatment failure. We used death from all causes as the end point for overall survival, which we also measured from the date of surgery. We analysed whether drug induced amenorrhoea in women menstruating at study entry was able to influence the outcome of treatment, excluding all patients who had a relapse within the first nine months after surgery. ${ }^{10}$ We used the Kaplan-Meier product limit method for all survival analyses. ${ }^{11}$ We used the log rank test to test the null hypothesis concerning the differential effects of treatment or of some prognostic factors in univariate analyses, ${ }^{12}$ and all $\mathrm{P}$ values were two tailed. In addition, we used a Cox regression model to investigate the joint effects of treatment and of prognostic indicators, ${ }^{13}$ using a backward selection procedure. We used Wald statistics to test the null hypothesis of the regression analysis. ${ }^{14}$ We estimated the relative risks as hazard ratios and calculated the rate of the sites of disease relapse as first event according to the method proposed by Marubini and Valsecchi. ${ }^{15}$ We analysed the data that were available as of 28 February 2003. Only two patients in complete clinical remission were lost to follow up, one after 15.8 years and the other after 20.7 years.

\section{Results}

\section{First CMF study}

Table 1 shows the summary of all four CMF studies carried out at our institute. In the first study, after a median follow up of 28.5 years and a minimal follow up of 25.4 years, both relapse free survival and overall survival (figure 1) remained significantly superior in women receiving adjuvant CMF than in women treated with surgery alone. As already reported, ${ }^{76}$ patients who received optimal doses of CMF ( $\geq 85 \%$ of the planned doses) showed a long lasting, superior benefit (relapse free survival $42 \%, 95 \%$ confidence interval $26 \%$ to $59 \%$; overall survival $40 \%$, $26 \%$ to $55 \%$ ) compared with patients who received lower doses (26\%, $19 \%$ to $33 \% ; 21 \%, 14 \%$ to $26 \%$ ). Table 2 shows the rates of relapse free and overall survival relative to main characteristics; no detrimental effect of adjuvant chemotherapy is shown for any of the subsets of patients. The lower rates of overall survival, especially in women aged 50 or older at study entry, can be explained by deaths not due to progression of breast cancer or new primary malignancies. They accounted for 22 events after surgery (median age at death 78 years, range 72-95) and for 24 events in the CMF group (median age at death 74 years, range 54-90).

The regression analyses of the joint effects of treatment and prognostic indicators confirmed the significant benefit of adjuvant chemotherapy $(\mathrm{P}=0.002$ for relapse free survival, $\mathrm{P}=0.04$ for overall survival). As reported in table $3, \mathrm{CMF}$ contributed to reducing the relative risk of disease relapse by $34 \%$ and of death from all causes by $22 \%$. The extent of nodal involvement remained a significant prognostic factor; patients with three or more positive nodes were also at an increased risk of relapse and death in this long term analysis. Neither age group nor menopausal status, oestrogen receptor status, or tumour size influenced relapse free survival significantly. As far as overall survival is concerned, patients aged 50 or more years at study entry had a significantly higher risk of dying (hazard ratio $1.43,95 \%$ confidence interval 1.12 to $1.82, \mathrm{P}=0.004$ ) than younger women.

Table 4 shows the cumulative incidence of first relapse according to anatomical sites. The main therapeutic effect of adjuvant $\mathrm{CMF}$ was to reduce the incidence of distant metastases (we found an absolute difference of $11 \%$ in our long term analysis between patients who received CMF and those who did not).

New malignancies other than contralateral breast cancers were documented in 12 patients (seven after surgery alone and 
Table 2 Relapse free and overall survival in the first CMF randomised study (enrolment June 1973 to September 1975). Median observation period 28.5 years

\begin{tabular}{|c|c|c|c|c|c|c|}
\hline \multirow[b]{2}{*}{ Characteristics } & \multicolumn{2}{|c|}{ No of patients } & \multicolumn{2}{|c|}{$\%$ relapse free ${ }^{*}$} & \multicolumn{2}{|c|}{$\%$ surviving* } \\
\hline & Surgery alone & CMF & Surgery alone & CMF & Surgery alone & CMF \\
\hline Total & 179 & 207 & 22 & 29 & 16 & 25 \\
\hline Premenopausal & 86 & 103 & 22 & 33 & 20 & 35 \\
\hline Postmenopausal & 93 & 104 & 23 & 26 & 11 & 14 \\
\hline \multicolumn{7}{|l|}{ Age: } \\
\hline$<50$ years & 75 & 95 & 21 & 30 & 21 & 35 \\
\hline$\geq 50$ years & 104 & 112 & 23 & 29 & 12 & 16 \\
\hline \multicolumn{7}{|l|}{ Tumour size: } \\
\hline$<2.0 \mathrm{~cm}$ & 96 & 103 & 26 & 31 & 16 & 27 \\
\hline$\geq 2.0 \mathrm{~cm}$ & 83 & 104 & 19 & 27 & 15 & 22 \\
\hline \multicolumn{7}{|c|}{ No of affected lymph nodes: } \\
\hline $1-3$ & 126 & 140 & 26 & 34 & 16 & 29 \\
\hline$>3$ & 53 & 67 & 14 & 20 & 15 & 16 \\
\hline \multicolumn{7}{|c|}{ Oestrogen receptor status:† } \\
\hline Negative & 51 & 54 & 25 & 31 & 19 & 26 \\
\hline Positive & 100 & 132 & 20 & 30 & 13 & 25 \\
\hline
\end{tabular}

${ }^{\star}$ Available for 337 of 386 patients (87\%).

†Percentage estimates are derived from the Kaplan-Meier product limit method.

five after CMF), with no prevailing distinctive pattern in either treatment group.

\section{CMF for six cycles compared with 12 cycles in}

\section{premenopausal patients}

After a median follow up of 25 years, the outcome of treatment was not improved with a longer duration of adjuvant CMF. The pattern of relapse free survival was the same in the two treatment groups, and the estimated, relapse free, survival rates were $39 \%$ after 12 cycles and 38\% after six cycles of CMF. At 25 years, the overall survival rates were $40 \%$ in both treatment arms. In the multivariate analysis, the only variable able to influence treatment outcome was the extent to which axillary nodes were affected; patients with three or more affected nodes had a significantly higher risk of disease relapse and death (hazard ratio 2.3, $95 \%$ confidence interval 1.61 to $3.16, \mathrm{P}=0.0001$ ). Neither oestrogen receptor status nor size of the primary tumour were able to affect therapeutic outcome significantly.

\section{CMF and amenorrhoea}

Grouping together all patients given CMF (first three studies in table 1), a total of 397 women had monthly periods before starting the 12 cycle regimen, and 145 had monthly periods before starting the six cycle regimen. In all these women, planned physical examinations included a diary of the women's monthly cycle. We defined drug induced amenorrhoea as the irreversible cessation of menstrual periods during chemotherapy treatment or in the first year of follow up, in the absence of disease relapse.
Table 5 reports the incidence of iatrogenic amenorrhoea in the two regimens by age group. Overall, drug induced amenorrhoea was reported more often in the longer regimen $(75 \% v 62 \%)$ than in the shorter one. However, in women aged 45 or older the incidence of amenorrhoea was unrelated to the duration of treatment $(97 \% v 96 \%)$.

To assess whether amenorrhoea induced by CMF could influence the outcome of treatment, we carried out an analysis that excluded all patients who had a relapse during the first nine months of chemotherapy. ${ }^{10}$ We selected this time period because in most women whose menstrual bleeds ceased irreversibly, this effect was observed during the first nine months, and because we wanted to allow comparisons between different studies. ${ }^{17-19}$ Figure 2 shows relapse free survival in patients who had monthly periods before starting 12 cycles of CMF and shows only a modest and non-significant advantage favouring patients with $\mathrm{CMF}$ induced amenorrhoea $(\mathrm{P}=0.2)$. A multivariate analysis including amenorrhoea, extent of nodal involvement, oestrogen receptor status, and age group confirmed that ovarian suppression induced by adjuvant CMF had no significant role in treatment outcome (hazard ratio $1.13,95 \%$ confidence interval 0.69 to 1.57 , $\mathrm{P}=0.6$ ); the only significant prognostic indicator remained the extent of nodal involvement. Results were similar in the 145 women allocated to receive six cycles of CMF (data not shown).

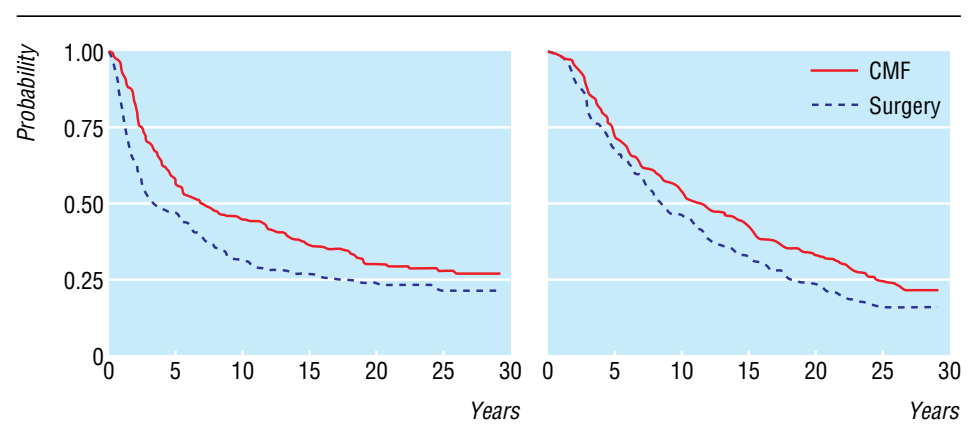

Fig 1 Treatment outcome in the first randomised CMF study after a median observation of 28.5 years. Left: Relapse free survival after surgery alone (179 patients) $V$ CMF (207 patients). Univariate analysis: hazard ratio 0.71 (95\% confidence interval 0.56 to $0.91 ; \mathrm{P}=0.005)$. Right: Overall survival after surgery alone (179 patients) $v$ CMF (207 patients). Univariate analysis: hazard ratio 0.79 (0.63 to $0.98 ; \mathrm{P}=0.04)$ 
Table 3 Multivariate analysis of the first CMF study in 337 patients with known oestrogen receptor status. Final model

\begin{tabular}{|c|c|c|c|}
\hline & Hazard ratio* & $95 \% \mathrm{Cl}$ & $P$ value (Wald test) \\
\hline \multicolumn{4}{|l|}{ Relapse-free survival: } \\
\hline $\begin{array}{l}\text { CMF } v \text { surgery } \\
\text { alone }\end{array}$ & 0.66 & 0.51 to 0.85 & 0.002 \\
\hline $\begin{array}{l}>3 \text { affected lymph } \\
\text { nodes } v 1-3 \\
\text { affected lymph } \\
\text { nodes }\end{array}$ & 1.67 & 1.28 to 2.18 & 0.0001 \\
\hline \multicolumn{4}{|l|}{ Overall survival: } \\
\hline $\begin{array}{l}\text { CMF } v \text { surgery } \\
\text { alone }\end{array}$ & 0.78 & 0.61 to 0.98 & 0.04 \\
\hline $\begin{array}{l}>3 \text { affected lymph } \\
\text { nodes } v 1-3 \\
\text { affected lymph } \\
\text { nodes }\end{array}$ & 1.40 & 1.09 to 1.80 & 0.009 \\
\hline $\begin{array}{l}\text { Age } \geq 50 v<50 \\
\text { years }\end{array}$ & 1.43 & 1.12 to 1.82 & 0.004 \\
\hline
\end{tabular}

${ }^{*}$ A ratio of $<1.0$ favours $\mathrm{CMF}$

\section{Intravenous $\mathrm{CMF}$ in node negative tumours}

As detailed in the original publications, ${ }^{9}{ }^{20}$ the randomised study comparing surgery alone with 12 cycles of the intravenous CMF regimen given every three weeks recruited only a limited sample of patients with node negative and oestrogen receptor negative tumours. After a median follow up of 19.2 years, our results confirm that CMF reduced the relative risk of both disease relapse and death by $35 \%$ (fig 3). Premenopausal and postmenopausal women benefited equally from adjuvant CMF, and small $(\leq 2.0$ $\mathrm{cm})$ and large tumours $(>2.0 \mathrm{~cm})$ were equally affected. $\mathrm{CMF}$ had the greatest effect on highly undifferentiated tumours (relapse free survival 32\% after surgery alone $v 63 \%$ after intravenous CMF), but it also affected differentiated tumours $(57 \%$ v 64\%).

\section{Discussion}

Our long term analysis of the trials we started three decades ago shows that the significant advantage in both relapse free and overall survival has persisted throughout the years and that adjuvant chemotherapy can suppress micrometastases to a moderate but worthwhile extent, regardless of their anatomical sites.

\section{Long term benefit of adjuvant chemotherapy}

Thirty years ago, treating patients who were free of identifiable metastatic disease with systemic adjuvant chemotherapy because some of them might eventually develop distant disease was a revolutionary departure from prior approaches to treatment. ${ }^{21}$ Improvements since the 1970s in the way breast cancer is managed are estimated to have prevented $25-30 \%$ of deaths in middle aged women who would otherwise have died from breast cancer in $2000 .^{22}$

\section{Benefit of CMF and menopausal status}

Although we observed no detrimental effect of adjuvant CMF as given in our studies, the magnitude of benefit of this regimen (overall reduction in the relative risk of relapse 29\%; overall reduction in the relative risk of deaths from all causes $21 \%$ after

Table 4 Cumulative incidence of first recurrence of cancer in the first CMF randomised study. Values are percentage estimates derived by applying the method of Marubini and Valsecchi

\begin{tabular}{|c|c|c|c|c|c|c|}
\hline \multirow[b]{2}{*}{ Recurrence } & \multicolumn{2}{|c|}{ At 5 years } & \multicolumn{2}{|c|}{ At 10 years } & \multicolumn{2}{|c|}{ In current analysis } \\
\hline & Surgery alone & CMF & Surgery alone & CMF & Surgery alone & CMF \\
\hline Total first recurrence: & 53 & 44 & 68 & 54 & 78 & 71 \\
\hline Locoregional only* & 12 & 10 & 14 & 12 & 15 & 14 \\
\hline Contralateral breast & 2 & 2 & 3 & 4 & 5 & 10 \\
\hline Distant & 39 & 32 & 51 & 38 & 58 & 47 \\
\hline
\end{tabular}

${ }^{*}$ Includes ipsilateral supraclavicular nodes

Table 5 Incidence of iatrogenic amenorrhea in premenopausal women who had monthly periods at study entry (first three studies in table 1)

\begin{tabular}{|c|c|c|c|c|}
\hline & \multicolumn{2}{|c|}{ CMF for 12 cycles } & \multicolumn{2}{|c|}{ CMF for 6 cycles } \\
\hline & No & No with amenorrhoea (\%) & No & No with amenorrhoea (\%) \\
\hline Total & 397 & $299(75)$ & 145 & $90(62)$ \\
\hline \multicolumn{5}{|c|}{ Age in years: } \\
\hline$<35$ & 48 & $6(12.5)$ & 8 & 00 \\
\hline $35-39$ & 87 & $52(60)$ & 38 & $11(29)$ \\
\hline $40-44$ & 110 & $94(85)$ & 50 & $32(64)$ \\
\hline$>44$ & 152 & $147(97)$ & 49 & $47(96)$ \\
\hline
\end{tabular}

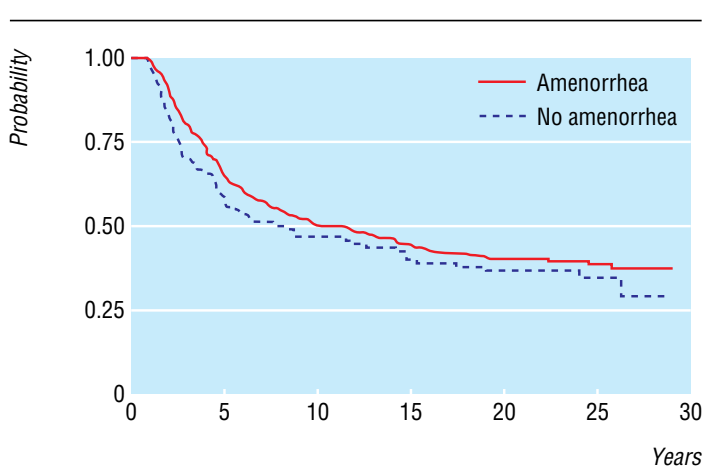

Fig 2 Relapse free survival in premenopausal women who had monthly periods at entry to the study and given 12 cycles of CMF. Influence of iatrogenic amenorrhoea 


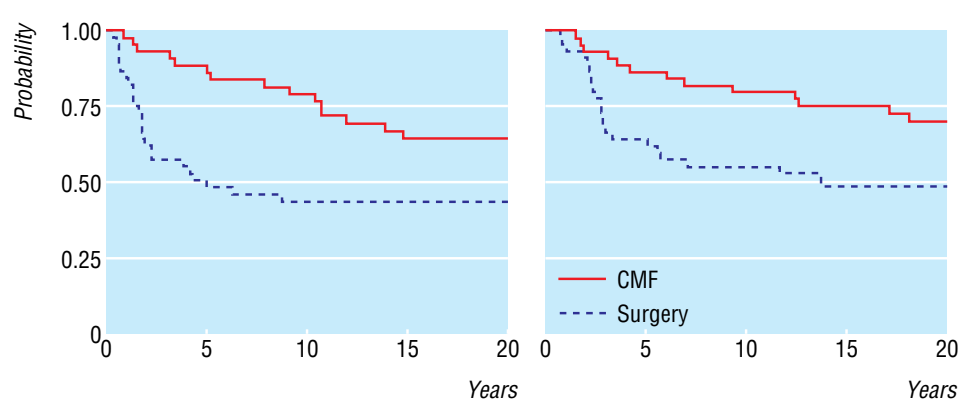

Fig 3 Treatment outcome in node negative and oestrogen receptor negative tumours: 20 year results. Left: Relapse free survival after surgery alone (45 patients) compared with intravenous CMF (45 patients). Univariate analysis: hazard ratio 0.65 (95\% confidence interval 0.47 to $0.90 ; P=0.009$ ). Right: Overall survival after surgery alone (45 patients) compared with intravenous CMF (45 patients). Univariate analysis: hazard ratio 0.65 ( 0.47 to $0.92 ; \mathrm{P}=0.01$ )

30 years) was apparently different between premenopausal and postmenopausal women. Although this different effect may be, at least in part, attributable to the lower doses of CMF delivered in women older than 60 years, ${ }^{2}{ }^{16}$ many investigators interpreted these results to mean that the predominant effect of chemotherapy was chemical castration. Recently released data from two randomised studies comparing the effects of CMF with endocrine manipulations in premenopausal women have reinforced this interpretation. ${ }^{5}{ }^{18-19}$ Our analysis of the influence of drug induced amenorrhoea on the therapeutic outcome after CMF treatment refutes the hypothesis that adjuvant chemotherapy acts merely as chemical castration. As reported in many individual trials (including our small study investigating node negative and oestrogen receptor negative tumours) and the worldwide overview, ${ }^{4}$ adjuvant chemotherapy benefits hormone responsive and hormone unresponsive tumours, whereas endocrine therapy has no worthwhile benefit in oestrogen receptor negative subpopulations. ${ }^{23}$ In addition, similar treatment results in a highly heterogeneous neoplastic disease such as breast cancer do not in themselves indicate that two different treatment modalities act through the same mechanism: our findings indicate that adjuvant chemotherapy has cytotoxic effects regardless of the putative hormone dependency of the tumour cells. The worldwide overview indicated that in hormone responsive tumours, the delivery of chemotherapy and endocrine therapy further reduces the relative risk of disease relapse and death compared with either modality alone. ${ }^{43}$

\section{Benefit of CMF and prognostic subsets}

Are there subpopulations with tumours in which effective adjuvant CMF does not achieve worthwhile benefits? The goal to tailor adjuvant treatment to characteristics of individual tumours, which is the subject of current trials, was inconceivable at the time when we designed our studies. However, the role of new biological variables, including c-erb-b2 expression, was retrospectively assessed in the first randomised trial comparing surgery alone with surgery and CMF in node positive cancers. ${ }^{24}$ In this trial, the poor prognosis associated with unfavourable indicators in the untreated group was overcome by adjuvant $\mathrm{CMF}$, and our analysis confirms these results.

\section{Conclusion}

Our findings from a long term follow up of a cohort of women participating in four separate trials between 1973 and 1985 confirm that the departure from conventional, locoregional treatment alone in operable breast cancer at risk of relapse, which we conceived and initiated 30 years ago, has contributed substantially to treatment and understanding of breast cancer today. The moderate but worthwhile therapeutic results achieved substantiate the recommendations of the consensus development conference of the National Institutes of Health in $2000^{25}$ on using chemotherapy outside clinical trials for patients with operable breast cancer at risk of disease relapse. New drugs available today include anthracyclines and taxanes, and these have improved outcomes of treatment over the CMF regimen. ${ }^{4}$ Although technological advances will further improve our understanding of breast cancer and will contribute to tailoring treatment to the individual patient, our experience with adjuvant CMF over 30 years confirms that the effects of such a regimen are long lasting and may benefit patients with favourable and unfavourable prognostic indicators, at the cost of minimal long term sequelae.

We thank all the patients who have participated in our clinical trials, and the many associates, in particular medical oncologists, surgeons, radiation therapists, pathologists, and research nurses, for their cooperation during the studies and follow up. We also thank George Canellos, Paul P Carbone, and Steve Carter for their invaluable advices during the planning and conduct of the studies.

Contributors: GB developed the protocols, secured initial funding, implemented the studies, and wrote the original draft. AM and MZ directed the clinical studies and helped with the final draft. MGD and SP directed the pathobiological studies and granted quality control for all assays. LG obtained long term funding, supervised the long term follow up, and helped with the final draft. PV organised the studies, assured the quality control of the data entry, carried out the statistical analysis, and wrote the original draft. GB, LG, PV are guarantors.

Funding: These studies were supported in part by contracts (N01-CM33714 and N01-CM-07338) with the Division of Cancer Treatment, National Cancer Institute, National Institutes of Health, Bethesda, Md.

Competing interests: None declared.

Ethical approval: Research and Ethics Committee of the Istituto Nazionale Tumori of Milan.

\section{What is already known on this topic}

At a median follow up of about 15 years, adjuvant systemic therapy with cyclophosphamide, methotrexate, and fluorouracil (CMF) can benefit patients with operable breast cancer

\section{What this study adds}

Adjuvant systemic therapy has long lasting effects even after 30 years, and these are achieved at the cost of minimal long term sequelae

The poor prognosis associated with unfavourable indicators in patients treated locoregionally alone was improved by administration of adjuvant CMF 
1 Bonadonna G, Brusamolino E, Valagussa P, Veronesi U. Adjuvant study with combination chemotherapy in operable breast cancer. Proc Am Assoc Cancer Res Am Soc Clin Oncol 1975;16:254

2 Bonadonna G, Brusamolino E, Valagussa P, Rossi A, Brugnatelli L, Brambilla C, et al Combination chemotherapy as an adjuvant treatment in operable breast cancer. $N E n g l$ J Med 1976;294:405-10.

3 Fisher B, Carbone P, Economou SG, Frelick R, Glass A, Lerner H, et al. l-Phenylalanine mustard (L-PAM) in the management of primary breast cancer: a report of early findings. N Engl J Med 1975;292:117-22.

4 Early Breast Cancer Trialists' Collaborative Group. Polychemotherapy for early breast cancer: an overview of randomised trials. Lancet 1998;352:930-42.

5 Pritchard KI. Adjuvant therapy for premenopausal women with breast cancer: is it time for another paradigm shift? J Clin Oncol 2002; 20:4611-4.

6 Ross JS, Fletcher JA. The HER-2/neu oncogene in breast cancer: prognostic factor, predictive factor, and target for therapy. Stem Cells 1998;16:413-28.

7 Bonadonna G, Valagussa P, Moliterni A, Zambetti M, Brambilla C. Adjuvant cyclophosphamide, methotrexate, and fluorouracil in node-positive breast cancer: the results of 20 years of follow-up. N Engl J Med 1995;332:901-6.

8 Tancini G, Bonadonna G, Valagussa P, Marchini S, Veronesi U. Adjuvant CMF in breast cancer: comparative 5-year results of 12 versus 6 cycles.J Clin Oncol 1983;1:2-10.

9 Zambetti M, Bonadonna G, Valagussa P, Daidone MG, Coradini D, Bignami P, et al. Adjuvant CMF for node-negative and estrogen receptor-negative breast cancer. J Nat Cancer Inst Monogr 1992;11:79-85.

10 Anderson JR, Cain KC, Gelber RD. Analysis of survival by tumor response. J Clin Oncol 1983;1:710-9.

11 Kaplan EL, Meier P. Nonparametric estimation from incomplete observations.J Am Stat Assoc 1958;53:457-81.

12 Peto R, Peto J. Asymptotically efficient rank invariant test procedures. J $R$ Stat Soc A 1972;135:185-207.

13 Cox DR. Regression models and life-tables (with discussion). J $R$ Stat Soc B 1972;34:187-220

14 Miller RG. Survival analysis. New York: John Wiley, 1981

15 Marubini E, Valsecchi M. Analysing survival data from clinical trials and observational studies. Chichester: John Wiley, 1995.

16 Bonadonna G, Valagussa P. Dose-response effect of adjuvant chemotherapy in breast cancer. N Engl J Med 1981;304:10-51.

17 Goldhirsch A, Gelber RD, Castiglione M. The magnitude of endocrine effects of adjuvant chemotherapy for premenopausal breast cancer patients. Ann Oncol 1990;1:183-8.
18 Jakesz R, Hausmaninger $\mathrm{H}$, Kubista $\mathrm{E}$, Gnant M, Menzel C, Bauernhofer T, et al Randomized adjuvant trial of tamoxifen and goserelin versus cyclophosphamide, methotrexate, and fluorouracil: evidence for the superiority of treatment with endocrine blockade in premenopausal patients with hormone-responsive breas cancer-Austrian Breast and Colorectal Cancer Study Group trial 5. J Clin Oncol 2002;20:4621-7.

19 Jonat W, Kaufmann M, Sauerbrei W, Blamey R, Cuzick J, Namer M, et al. Goserelin versus cyclophosphamide, methotrexate, and fluorouracil as adjuvant therapy in premenopausal patients with node-positive breast cancer: the Zoladex early breast cancer research association study. J Clin Oncol 2002; 20:4628-35.

20 Zambetti M, Valagussa P, Bonadonna G. Adjuvant cyclophosphamide, methotrexate, and fluorouracil in node-negative and estrogen receptor-negative breast cancer. Ann Oncol 1996;7:481-5.

21 Fisher B. The evolution of paradigms for the management of breast cancer: a personal perspective. Cancer Res 1992;52:2371-83.

22 Peto R, Boreham J, Clarke M, Davies C, Beral V. UK and USA breast cancer deaths down 25\% in year 2000 at ages 20-69 years. Lancet 2000;355:1822.

23 Early Breast Cancer Trialists' Collaborative Group. Tamoxifen for early breast cancer: an overview of the randomised trials. Lancet 1998;351:1451-67.

24 Ménard S, Valagussa P, Pilotti S, Gianni L, Biganzoli E, Boracchi P, et al. Response to CMF in lymph-node positive breast cancer according to HER2 overexpression and other tumours biologic variables. J Clin Oncol 2001:19:329-35.

25 Eifel P, Axelson JA, Costa J, Crowley J, Curran WJ Jr, Deshler A, et al. National Institutes of Health Consensus Development Conference Statement: adjuvant therapy for breast of Health Consensus Development Conference Statement: adj
cancer, November 1-3, 2000. J Natl Cancer Inst 2001;93:979-89.

(Accepted 15 November 2004)

doi $10.1136 /$ bmj.38314.622095.8F

Istituto Nazionale Tumori, 20133 Milan, Italy

Gianni Bonadonna consultant, Department of Medical Oncology

Angela Moliterni senior staff, Division of Medical Oncology A

Milvia Zambetti senior staff, Division of Medical Oncology A

Maria Grazia Daidone director, Determinant of Prognosis and Treatment Response Unit

Silvana Pilotti director, Division of Pathology $C$

Luca Gianni director, Division of Medical Oncology A

Pinuccia Valagussa head, Operations Office, Department of Medical Oncology

Correspondence to: G Bonadonna gianni.bonadonna@istitutotumori.mi.it 